\title{
Performance Evaluation of Routing Protocol for FANET Using Ns2
}

\author{
Megha N \\ Student, M.Tech-Digital communication engineering \\ Department of Electronics and Telecommunication \\ Engineering \\ Siddaganga Institute of Technology, Tumakuru \\ Karnataka, India
}

\author{
Dr. Mallikarjun B C \\ Associate Professor \\ Department of Electronics and Telecommunication \\ Engineering \\ Siddaganga Institute of Technology, Tumakuru \\ Karnataka, India
}

\begin{abstract}
A Flying Ad-hoc Networks (FANETs) is a network that comprises a community of Small, Ad-hoc-connected UAVs which are part of a team to hit Goals at the highest point. In recent technologies as in the field of research and development, FANETs have become more popular. In FANETs, routing protocols play a critical role during the endto - end data transmission between UAV to UAV. FANETs can be used to provide fast, scalable, self-configured networks. There are so many existing routing protocols and each protocol has its own disadvantages and drawbacks. The main purpose here is to evaluate the various routing protocols such as AODV, DSDV, DSR, OLSR based on parameters such as Total packet loss, Throughput, End to End delay over number of nodes.
\end{abstract}

Keywords: Flying Ad-Hoc Network, Unmanned Aerial Vehicle, Network Simulator, AODV, DSDV, DSR, OLSR.

\section{INTRODUCTION}

Progress in recent technology leads to autonomous flying machines like Drones, UAVs that can operate practically without human interference.

The autonomous flying machines can be used in military applications to track enemies and their movements and continuous border monitoring operations, etc., Medical applications for transplantation of drugs and even organs, Agriculture applications for the spare of pesticides and insecticides and observation of plants and fields in real time, Forestry applications for fire detection, animals etc.

Unmanned aerial vehicle (UAV) systems can operate independently or can run remotely. UAVs are commonly referred to as Drones. UAVs are designed for autonomous flight and remotely piloted vehicles that operate remotely by a ground control operator. UAVs are fitted with a micro-controller for processing input commands and can be remotely controlled from outside and fitted with wireless transceivers for communicating with other UAVs or ground-based units. Within a UAV all nodes are linked to a ground station via single or multihop communication. A UAV network includes wireless system for data sending, GPS unit for location monitoring, cameras for image and video recording, various sensors for desire parameter sensing, etc. UAV use is on the rise day by day.

Single-UAV systems have been used for a long time, but nowadays the re-search and deployment has moved to
multi-UAV system applications. Using a single-UAV system is very

popular, but it has become beneficial to use a group of UAVs,

such as Multi-UAVs are more affordable, Versatile, Quicker,

accuracy, Reliable and easy to predict troubles. There are some conflicts with Multi-UAV systems. A ground base station or satellite is being used for communication within a single-UAV network. Often, contact between the UAV and an airborne control system is provided. In every case, single-UAV communication link is established between the $\mathrm{UAV}$ and the infrastructure. When the number of UAVs increases in the unmanned aerial systems, designing effective network architectures becomes a crucial issue. A further issue is the limitation of range between the UAVs and the ground base station. If a UAV is outside the ground station's coverage area it gets detached. To solve the problems, the creation of an Ad-Hoc network among UAVs, called as FANETs, requires an alternative solution. A subgroup of UAVs can link up with the ground station or the satellite in FANETs and all UAVs are an Ad-Hoc network work. In this manner the UAVs, besides the ground station, can communicate with each other.

\section{A. Objective}

1. To study about different routing protocols and analyze the routing protocols for different parameters.

2. To compare a performance of different routing protocols.

3. To implement a routing protocol to FANETs.

\section{RELATED WORKS}

In the paper [1], Analyze and tested different routing protocols to choose the best protocol for FANETs on the packet delivery ratio, End to End delay and Throughput. In results, HWMP has been recognized as best protocol and OLSR occupies the second spot in terms of overall performance. In simulation scenarios, analyzed that sometimes OLSR, HWMP performance goes hand in hand and sometimes overheads in OLSR are less as compared to HWMP. First introduced three different decentralized communication architectures, and then proposed a multi- 
layer UAV Ad-Hoc network more suitable for FANETs. Subsequently, investigated various routing protocols along with open research issues and this investigation will help network engineers for FANETs deployment[2].

In this paper[3], analyzed and compared the performance of two routing protocols: AODV and DSDV in a FANET. The results obtained are possible to appreciate good performances and slight differences between the protocols choose. A notable difference between them can be observed moving the speed of the nodes. Here from the results, it's recommended the use of the AODV routing protocol for the proposed FANET (UAV network of four nodes).

In this paper[4], AODV, DSDV, and OLSR routing protocols are analyzed under the different parameters like End to END delay, Average Throughput and Packet delivery ratio with respect to speed of mobile node. Through the simulation results it can be clearly seen that, OLSR routing protocol perform better than the other two routing protocol AODV and DSDV. So the performance of FANET can be optimized by choosing OLSR as a routing protocol.

In this paper[5], compared the performance of P-OLSR and that of OLSR in a FANET. Such networks are characterized by a high degree of mobility, which continues a challenge to the routing protocol. Routing protocols designed for MANETs mostly fail in tracking the evolution of the network topology. This problem is address by designing an OLSR extension called P-OLSR. It takes advantage of the GPS information to predict how the quality of the wireless links will evolve. With P-OLSR, the routing follows the topology changes without interruptions, which is not the case with OLSR.

Communication is one of the most challenging issues for multi-UAV systems. Multi-UAV system reduces the operation accomplishment time and increases reliability of the system for airborne operations when compared to a single-UAV system. To apply networking in non-LOS, urban, aggressive and noisy environment multi-UAV system is very effective and accurate. In this paper, AdHoc networks among the UAVs, i.e., FANETs are surveyed along with its key challenges compared to traditional Ad-Hoc networks. The exiting routing protocols for FANETs are classified into six major categories which are then critically analyzed and compared based on various performance criteria[6].

\section{PROPOSED SYSTEM}

The best routing protocol is defined; by analyze the performance of different Routing protocols based on different parameters like Packet loss, End to End delay, throughput etc.

A. Performance parameters:

Performance parameters are used to compare the different routing protocols.

Some performance parameters are:

\section{Total packets loss:}

Packet loss=generated packets-received packets

\section{End-to-End Delay:}

Average amount of time taken by a packet to go from source to destination.

Delay=receiving time-sending time

Average delay=Delay/count

\section{Throughput:}

It is defined as total number of packets successfully received by the destination.

Throughput in Mbps=received data * 8/data transmission period.

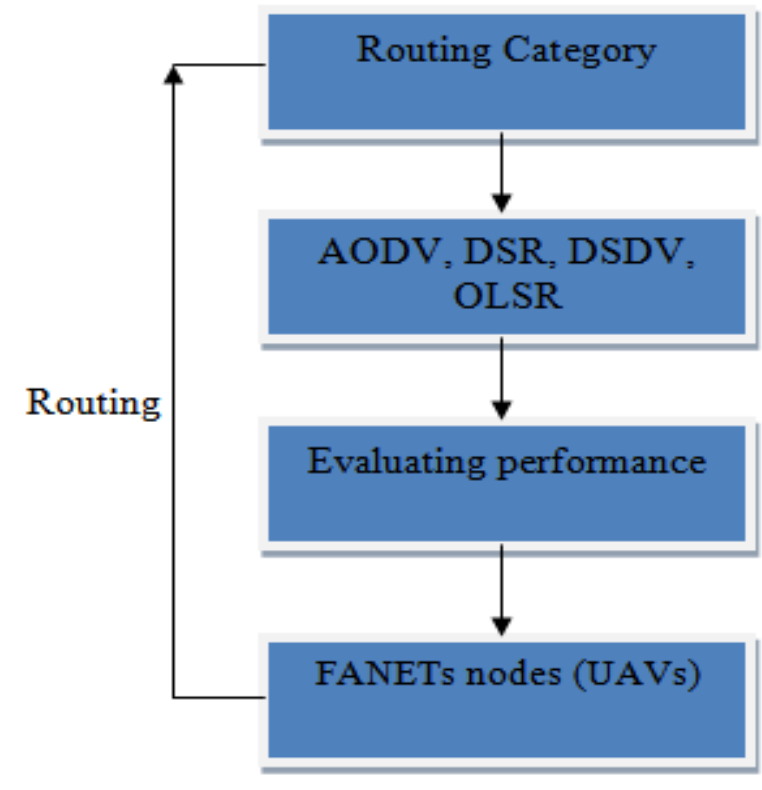

Fig.1: Flow chart

\section{B. FANETs}

FANETs are such a type of network, consisting of a number of small ad-hoc-connected UAVs that are assembled into a team to achieve high-level objectives. The key aspect of FANETs is mobility, shortage of centralization, self-organization and Ad-Hoc interaction between each of the UAVs.

Flying Ad-Hoc Networks is a new variant of Ad-Hoc networks, usually consisting of homogeneous or heterogeneous flying objects (UAV / Drones) which communicate, interact and coordinate with each other to grab data through sensors. FANETs are considered to be ideal networks for delivering information smartly and easily comparable to Ad-Hoc networks in so many situations including Flood areas, Earthquake affected area, Battlefields, Mountain Eruption condition tracking and others. Each FANETs node has to be fitted with powerful hardware to communicate with ground server or satellite for improved service. A further difficulty in FANET is Accuracy in Communication.

FANETs do not have a core infrastructure; so they are very durable towards individual threats or network breakdown. Furthermore, as these networks focus on no external assistance, they can be dispatched easily anywhere. On the one hand, these features make FANETs the most 
convenient approach for several tasks, but on the other hand, they present a complicated networking issue. In fact, the configuration of a FANET can differ drastically due to the rapid and irregular motion of the UAVs, and the node must respond by instantly upgrading its routing tables. Hence, implementing a reliable and sensitive routing protocol is essential in FANETs.

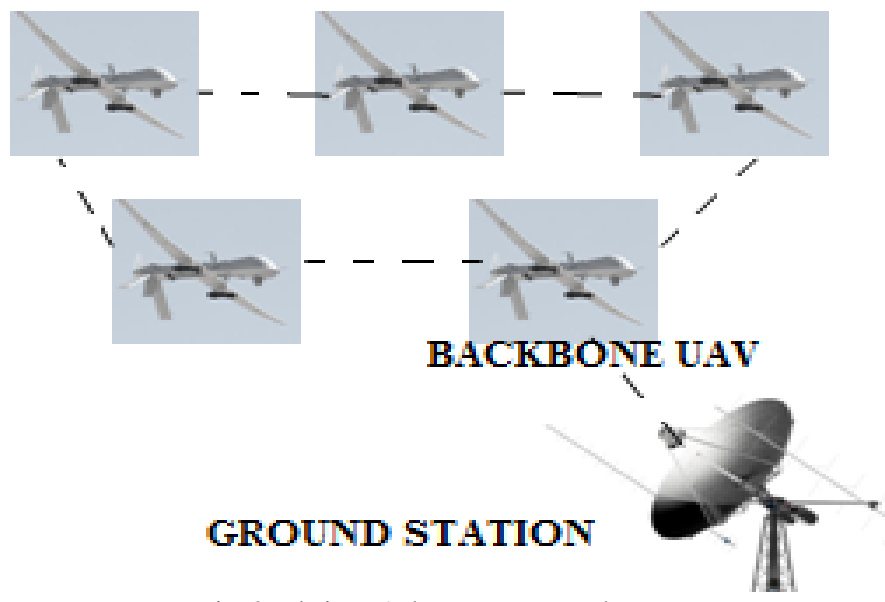

Fig.2:Flying Ad-Hoc Network

\section{ROUTING PROTOCOLS}

Whenever a packet wants to be transmitted via different nodes in the network, a routing protocol is required. All such protocols find a suitable route to deliver the packets to the proper destination. The performance of FANETs has to do with routing protocol capacity. Performance focuses on many considerations, such as convergence time after variations in topology, bandwidth overhead to allow appropriate routing and power requirements.

The different classifications of routing protocols are: Proactive, Reactive and Hybrid protocols.

Proactive protocol: In these types of routing protocol, every node retains routing information about the other nodes within the network via the routing tables, which are changed regularly as the topology of the network changes.

The different types of proactive protocols are: Destination Sequenced Distance Vector (DSDV), Wireless Routing Protocol (WRP), Fish eye State Routing Protocol (FSR), Optimised Link State Routing Protocol (OLSR), Cluster Gateway Switch Routing protocol(CGSR), Topology Dissemination Based on Reverse Path Forwarding (TBRPF), etc.

Reactive protocol: The routes are generated when they are required. While the destination is reachable or until the route is no longer required, the route remains valid. This kind of routing protocol is best suited for FANETs but this form of routing results in high latency and there is no routing protection.

The different types of Reactive protocols are: Ad-Hoc ON Demand Distance Vector (AODV), Dynamic Source Routing Protocol (DSR), Temporally Ordered Routing Algorithm (TORA), Associativity Based Routing (ABR), etc.

Hybrid protocol: To overcome the proactive routing protocols routing overhead problems and reactive protocols high latency problem, the hybrid protocol is proposed. The different types of hybrid protocols are Hybrid Wireless Mesh Protocol (HWMP),Zone Routing protocol (ZRP), Secured Hierarchical Anonymous Routing Protocol (SHARP), Temporally Ordered Routing Algorithm (TORA), etc.

The following protocols for performance analysis are discussed in this research paper: AODV, DSDV, DSR and OLSR on parameters such as packet loss, End to End delay, Throughput.

\section{Ad-Hoc On Demand Distance Vector (AODV):}

AODV a reactive routing protocol is designated as one of the finest routing protocols with outstanding flexibility to cope to rapidly changing communication demands, use low processing and lower overhead memory, low network consumption and efficient of determine unicast routes from source node to destination node with loop avoidance. AODV routing protocol comprises of three phases:

(i) Route discovery

(ii) Packet Transmitting

(iii) Route Maintaining.

If a source UAV wants to send a packet, it initiates a route discovery operation first to detect the location of the intended UAV and then forward the packet over a specified route without making a loop during the packet transmission process. The maintenance process of the route is performed to recover link failure.

\section{Destination Sequenced Distance Vector (DSDV):}

The DSDV routing protocol will be used for FANET routing. Each aerial vehicle must have all the details about other aerial vehicles functioning in the network within that protocol. This protocol that uses the sequence number assigned to the Destination node to resist looping and network congestion when there is any change in the topology occurs. Every UAV with a stronger sequence number is faster and more efficient than the low UAV sequencing number.

The routing table is maintained in this protocol which includes three parameters like Destination, Distance and Next Hop. Each node transmits the routing table at a specific interval nearby nodes and other nodes recalculate the parameters. DSDV has been designed to limit the distance vector routing restrictions and add to them two route parameters: sequence number, damping.

\section{Dynamic Source Routing (DSR):}

The DSR protocol is a reactive, wireless multi-hop routing protocol, that is also used for the routing of data packets between UAVs in the FANETs. UAV sends the data packet with a Request ID under the following protocol to prevent congestion of any kind. Under DSR, each source node saves the path from its position in the data header to its destination, where any kind of network problem like the connection Failure is performed in order to create new paths. 


\section{Optimized Link State Routing (OLSR):}

Each node has full information concerning neighboring nodes in OLSR's routing protocol. Two types of messages are sent over the network in this protocol. One is 'Hello' to check the connection to nearby nodes at 1-hop distance at regular intervals. The second message is 'control' message used to communicate information about some kind of adjustment to the network. For example, sometimes a lot of overhead can be faced with a message. Therefore, the Directional Optimized Link State Routing Protocol (DOLSR) is recommended for overcoming the overhead problem.

\section{IV.SIMULATION}

\section{A. Simulation Platform:}

NS2 was developed by the University of California and Cornell University, an open-source platform for discrete event simulating, in 1989. It uses the language $\mathrm{C}++$ and OTcl language for programming and it is purely based on object oriented programming language.

NS-2.35 version is used to measure the performance and simulation of FANETs Routing protocols.

\section{B. Simulation Parameters:}

\begin{tabular}{|l|l|}
\hline Simulation parameter & Values \\
\hline Simulator and version & $\begin{array}{l}\text { Ns-all-in-one(version } \\
2.35)\end{array}$ \\
\hline Channel Type & Wireless \\
\hline Protocols used & $\begin{array}{l}\text { AODV, DSDV, DSR, } \\
\text { OLSR }\end{array}$ \\
\hline Number of UAVs & 100 \\
\hline Type of Traffic & CBR \\
\hline Mac Layer Protocol & 802.11 \\
\hline
\end{tabular}

\section{Simulation Results:}

The output of Simulation of AODV in NS2 for 100 is shown in Fig.3

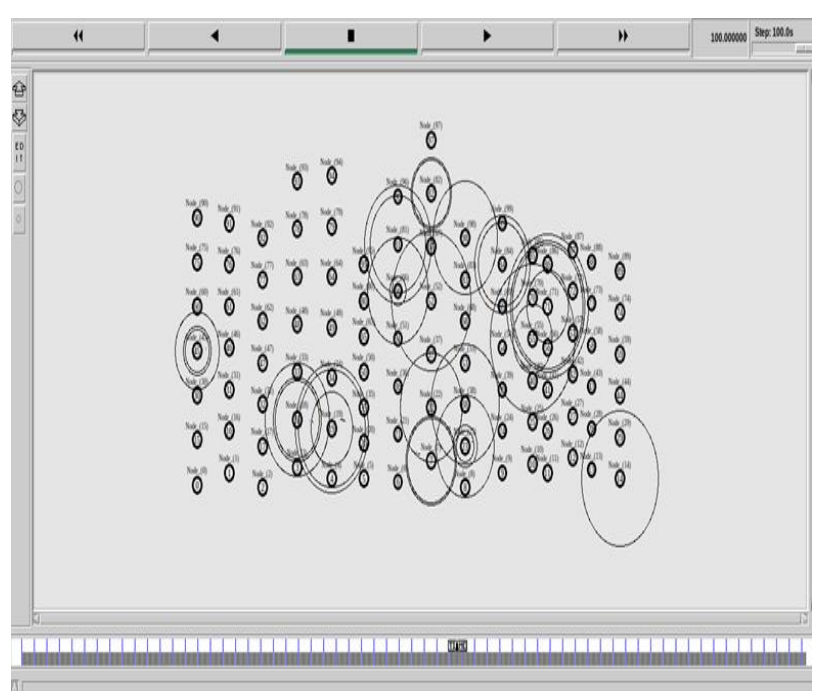

\section{i. Total Packets Loss:}

In NS-2, simulation is performed and the corresponding output for total packets loss as shown,

\begin{tabular}{|l|l|l|l|l|l|}
\hline \multirow{2}{*}{$\begin{array}{l}\text { Routing } \\
\text { Protocol }\end{array}$} & \multicolumn{5}{|c|}{ Numbers of Nodes } \\
\cline { 2 - 6 } & 10 & 30 & 50 & 70 & 90 \\
\hline AODV & 0 & 2 & 2 & 3 & 4 \\
\hline DSDV & 0 & 3 & 4 & 7 & 11 \\
\hline DSR & 0 & 1 & 1 & 2 & 2 \\
\hline OLSR & 0 & 2 & 3 & 2 & 3 \\
\hline
\end{tabular}

Table 2: Total packets loss

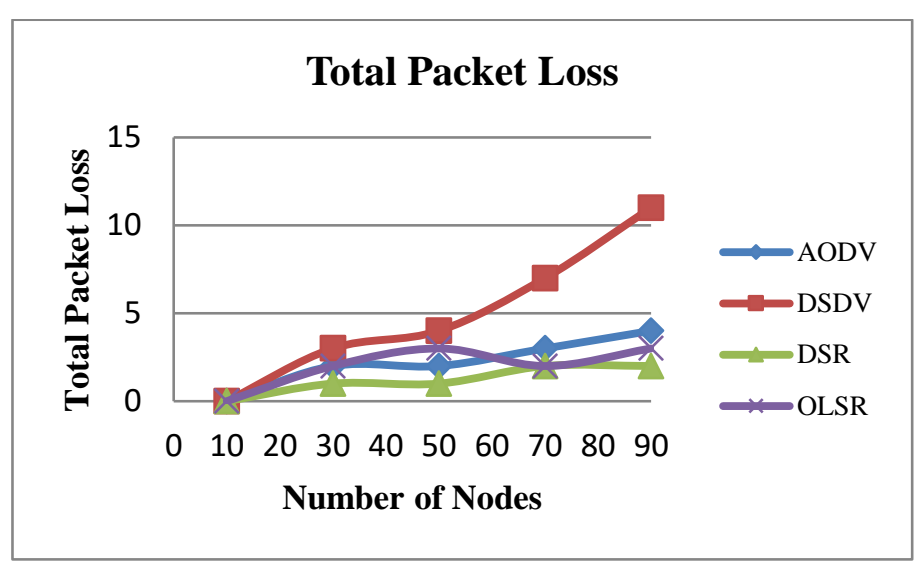

Fig. 4: Graphical Representation of Total Packets loss

\section{ii. End-To-Delay:}

In NS-2, simulation is performed and the corresponding output for End-To-Delay as shown,

\begin{tabular}{|l|r|r|r|r|r|}
\hline Routing & \multicolumn{5}{|c|}{ Number of Nodes } \\
\cline { 2 - 6 } 1 & 10 & 30 & 50 & 70 & 90 \\
\hline AODV & 0.092 & 4.1928 & 10.088 & 12.186 & 14.992 \\
& 4 & 2 & 4 & 4 & 4 \\
DSDV & 0.082 & 4.0828 & & 12.081 & 14.126 \\
\hline & 4 & 2 & 9.0924 & 2 & 9 \\
DSR & 0.295 & & & & \\
\hline & 6 & 4.5282 & 10.98 & 13.629 & 14.092 \\
OLSR & 0.082 & & 10.386 & & 13.925 \\
& 4 & 4.0032 & 2 & 11.691 & 2 \\
\hline \multicolumn{7}{|c|}{ Table 3: End-To-Delay } \\
\end{tabular}

Fig.3: Simulation of AODV in NS2 


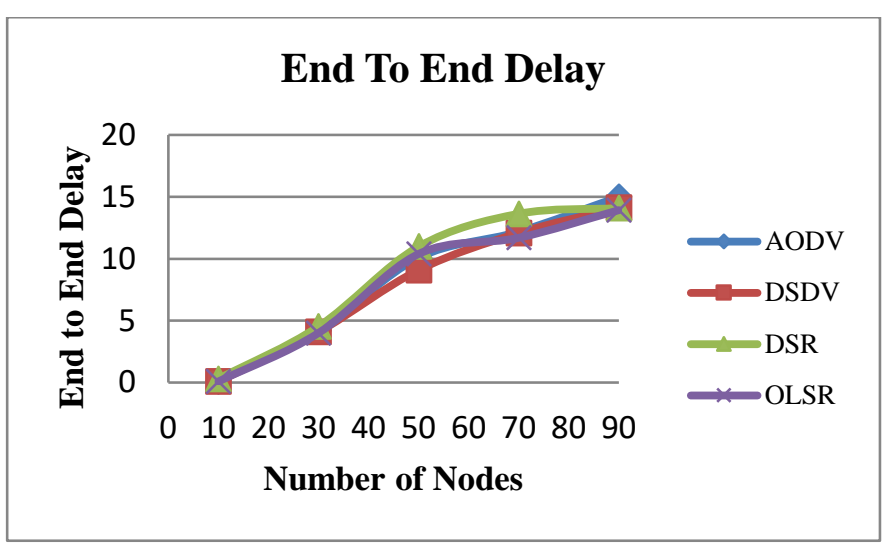

Fig. 5: Graphical Representation of End To End Delay

\section{iii. Throughput}

In NS-2, simulation is performed and the corresponding output for throughput as shown,

\begin{tabular}{|l|c|c|c|c|c|}
\hline \multirow{2}{*}{$\begin{array}{l}\text { Routing } \\
\text { Protocol }\end{array}$} & \multicolumn{5}{|c|}{ Numbers of Nodes } \\
\cline { 2 - 6 } & 10 & 30 & 50 & 70 & 90 \\
\hline AODV & 0.092 & 4.1928 & 10.088 & 12.186 & 14.992 \\
\hline DSDV & 0.082 & 4.0828 & 9.0924 & 12.0812 & 14.1269 \\
\hline DSR & 0.2956 & 4.5282 & 10.98 & 13.629 & 14.092 \\
\hline OSR & 0.0824 & 4.0032 & 10.3862 & 11.691 & 13.9252 \\
\hline
\end{tabular}

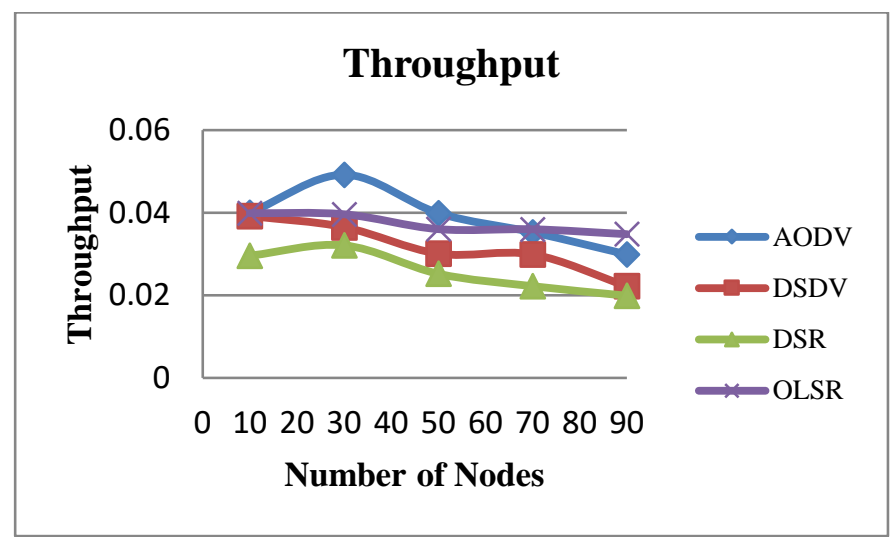

Fig. 6: Graphical Representation of Throughput

\section{CONCLUSION}

For FANETs, to choose a best routing protocol, the performance is analysed over different routing protocols like AODV, DSDV, DSR, and OLSR on the bases of performance parameter like Total packet loss, End-to-End delay and Throughput. From overall results OLSR has been recognized as best routing protocol among AODV, DSDV and DSR.

\section{REFERENCES}

[1] A. Nayyar, "Flying Adhoc Network (FANETs): Simulation Based Performance Comparison of Routing Protocols: AODV, DSDV DSR, OLSR, AOMDV and HWMP," 2018 International Conference on Advances in Big Data, Computing and Data Communication Systems (icABCD), Durban, 2018, pp. 1-9.

[2] M. A. Khan, A. Safi, I. M. Qureshi and I. U. Khan, "Flying adhoc networks (FANETs): A review of communication architectures and routing protocols," 2017 First International Conference on Latest trends in Electrical Engineering and Computing Technologies (INTELLECT), Karachi, 2017, pp. 1-9.

[3] Garcia-Santiago, J. Castaneda-Camacho, J. F. GuerreroCastellanos and G. Mino-Aguilar, "Evaluation of AODV and DSDV routing protocols for a FANET: Further results towards robotic vehicle networks," 2018 IEEE 9th Latin American Symposium on Circuits \& Systems LASCAS), Puerto Vallarta, 2018, pp. 1-4.

[4] K. Singh and Anil Kumar Verma, "Experimental analysis of AODV, DSDV and OLSR routing protocol for flying adhoc networks (FANETs)," 2015 IEEE International Conference on Electrical, Computer and Communication Technologies (ICECCT), Coimbatore, 2015, pp. 1-4.

[5] S. Rosati, K. Krużelecki, G. Heitz, D. Floreano and B. Rimoldi, "Dynamic Routing for Flying Ad Hoc Networks," in IEEE Transactions on Vehicular Technology, vol. 65, no. 3, pp. 16901700, March 2016

[6] M. H. Tareque, M. S. Hossain and M. Atiquzzaman, "On the routing in Flying Ad Hoc Networks," 2015 Federated Conference on Computer Science and Information Systems (FedCSIS), Lodz, 2015, pp. 1-9.

[7] Dr. L. Raja. "Performance Evaluation of AODV Protocol Using NS2 Simulator" IJFRCSCE July 2018, Available@ http://www.ijfrcsce.org

[8] S. Mohseni, R. Hassan, A. Patel, and R. Razali, "Comparative Review Study of Reactive and Proactive Routing Protocols in MANETs". ,4th IEEE International Conference on Digital Ecosystems and Technologies, 2010, pp.304-309.

[9] Perkins, C., Belding-Royer, E., \& Das, S. (2003). "Ad hoc on demand distance vector (AODV) routing” (No. RFC 3561).

[10] Marina, M. K., \& Das, S. R. (2001, November). "On-demand multipath distance vector routing in ad hoc networks". In Network Protocols, 2001. Ninth International Conference on(pp 14-23). IEEE.

[11] Narra, H., Cheng, Y., Cetinkaya, E. K., Rohrer, J. P., \& Sterbenz, J. P. (2011, March). "Destination-sequenced distance vector (DSDV) routing protocol implementation in ns-3". In Proceedings of the 4th International ICST Conference on Simulation Tools and Techniques (pp. 439-446). ICST (Institute for Computer Sciences, Social-Informatics and Telecommunications Engineering).

[12] Johnson, D. B., Maltz, D. A., \& Broch, J. (2001). "DSR: The dynamic source routing protocol for multi-hop wireless ad hoc networks". Ad hoc networking, 5, 139-172.

[13] Clausen, T., Hansen, G., Christensen, L., \& Behrmann, G. (2001, September). "The optimized link state routing protocol, evaluation through experiments and simulation". In IEEE Symposium on Wireless Personal Mobile Communications. 